\title{
LA FORMACIÓN PROFESIONAL EN EL EMPLEO DOMÉSTICO: ANÁLISIS DEL ESTADO DE LA CUESTIÓN
}

\section{Vocational training in domestic employment: analysis state of the question}

\author{
Carmen Grau Pineda*
}

Universidad de Las Palmas de Gran Canaria, España

\begin{abstract}
RESUMEN
El sector del empleo doméstico es un observatorio privilegiado que evidencia la vigencia de la división sexual del trabajo clásica que descansa sobre la explotación laboral de las personas trabajadoras del sector y perpetúa la discriminación por razón de sexo en un mercado laboral profundamente segmentado como el espańol. $Y$ es evidente que, frente a ello, es necesario tanto fomentar la corresponsabilidad intrafamiliar como incentivar la formación y cualificación del colectivo y los servicios que presta, fundamentales, ineludibles en el nuevo contexto social que la incorporación de las mujeres al mercado de trabajo dibuja en el presente y, como no, en el futuro inmediato. Se está en presencia de un sector emergente, un yacimiento de empleo, un servicio esencial a la comunidad y una actividad cuya demanda seguirá una tendencia creciente ante la falta de provisión pública de cuidados y que, por el bien de todos, es preciso replantear de forma inminente en la siguiente línea: dignificarla y profesionalizarla. Y para ello el papel de la formación y la cualificación profesional es innegable se mire por donde se mire.

Palabras clave: trabajo doméstico, formación profesional, mujeres, cualificación, dignificación.
\end{abstract}

\section{ABSTRACT}

The domestic employment sector is a privileged observatory that evidences the validity of the classic sexual division of labor that rests on the labor exploitation of substitutes or servants and perpetuates discrimination in a deeply segmented labor market. And it is evident that, in the face of this, it is necessary both to promote intra-family co-responsibility and to encourage the training and qualification of the group and the services it provides, fundamental, unavoidable in the new social context that the incorporation of women into the labor market draws in the present and, of course, in the immediate future. We are in the presence of an emerging sector, a source of employment, an essential service to the community and an activity whose demand will continue to grow in the face of the lack of public provision of care and that, for the good of all, it is necessary to rethink imminent form in the following line: dignify and professionalize it. And for this, the role of training and professional qualification is undeniable no matter where you look.

Keywords: domestic work, professional training, women, qualification, dignity.

1 ORCID: 0000-0003-0274-8875 y ResearchID: N-9223-2014. Este trabajo se encuadra en las actividades del Laboratorio de Derecho Social, línea de estudio Trabajo Doméstico y del Seminario Permanente de Trabajo Doméstico de la UC3M, Proyecto subvencionado en la convocatoria 2019, IMIO, 12/4ACT, ambos dirigidos por la profesora M. ${ }^{\mathrm{a}}$. Gema QUINTERO LIMA. Es éste el resultado avanzado de una investigación que se presentó en formato póster en el III Congreso Mundial CIELO LABORAL «Quel Rôle pour les entreprises, les travailleur, les organisations représentatives, les citoyens et les États dans le Droit du travail et de la protection sociale du 21ème siècle?», 24-25 septiembre de 2020.

* Correspondencia a: Carmen Grau Pineda. Paseo San Antonio, 57, a2 (Las Palmas de Gran Canaria), España - carmen.grau@ulpgc.es - https://orcid.org/0000-0003-0274-8875

Cómo citar: Grau Pineda, Carmen. (2020). «La formación profesional en el empleo doméstico: análisis del estado de la cuestión»; Lan Harremanak, 44, 358-378. (https://doi.org/10.1387/lan-harremanak.22195).

Recibido: 26 octubre, 2020; aceptado: 18 noviembre, 2020.

ISSN 1575-7048 - elSSN 2444-5819 / (c) 2020 UPV/EHU

(c) (i) Esta obra está bajo una licencia

Creative Commons Atribución 4.0 Internacional 


\section{1. ¿Existe un derecho laboral a la formación profesional para las personas trabajadoras del sector del empleo doméstico?}

En España, hoy por hoy, el empleo doméstico se considera un sustituto de la atención institucional. Y es que en un sector como éste caracterizado por la escasa implicación de las administraciones públicas (frente a otras opciones europeas muchos más institucionalizadas) y la ausencia de una respuesta eficaz por parte del mercado privado, las familias se ven impulsadas a buscar otras alternativas a través de la contratación informal de mujeres, en muchos casos, inmigrantes que, a menor coste y con gran disponibilidad, están dispuestas a realizar trabajos rechazados por las mujeres autóctonas.

De todo ello, puede colegirse que ha sido la presencia de mujeres inmigrantes la que ha terminado por consolidar, por tanto, la "feminización" del sector en ambos lados de la relación laboral. Es decir, «aunque parte de las actividades domésticas se han mercantilizado, continúan siendo "cosas de mujeres» tanto por quién las realiza como por quién las contrata y gestiona su trabajo. El trabajo reproductivo sigue atribuyéndose a las mujeres, sin cuestionar las relaciones patriarcales en la sociedad que apenas cambian en el ámbito privado ante la falta de compromiso e implicación de los varones en él. De poco sirve que la ley garantice la igualdad formal si, diseñadas las políticas públicas en términos de igualdad, su implementación práctica demuestra, con datos empíricos, que las usuarias/beneficiarias de las mismas siguen siendo las mujeres mayoritariamente dado que los varones se sienten al margen de todo lo relacionado con los cuidados y el hogar familiar².

Por todo ello, este sector es un observatorio privilegiado que evidencia la vigencia de la división sexual clásica del trabajo que descansa sobre la explotación laboral de las sustitutas o servidoras y perpetúa la discriminación en un mercado laboral profundamente segmentado (Grau, 2020: 103 y ss.). Y es evidente que, frente a ello, es necesario tanto fomentar la corresponsabilidad intrafamiliar porque la infravaloración del trabajo femenino conduce a su desprofesionalización como incentivar la formación y cualificación del colectivo y los servicios que presta, fundamentales, ineludibles en el nuevo contexto social que la incorporación de las mujeres al mercado de trabajo dibuja en el presente y, como no, en el futuro inmediato.

2 Interesa precisar desde estas primeras líneas que, pese a tratarse de conceptos diferentes, es frecuente que se utilicen de forma complementaria porque, en la práctica, el trabajo doméstico incluye cuidados de personas no dependientes. Mientras que el cuidado de personas dependientes (reconocimiento de la situación de dependencia y del derecho a las prestaciones del Sistema para la Autonomía y Atención de la Dependencia — SAAD—) se cubre por otras vías en las que no procede entrar en esta aportación. 
Se está en presencia de un sector emergente, un yacimiento de empleo, un servicio esencial a la comunidad y una actividad cuya demanda seguirá una tendencia creciente ante la falta de provisión pública de cuidados y que, por el bien de todos, es preciso replantear de forma inminente en la siguiente línea: dignificarla y profesionalizarla. Y para ello el papel de la formación y la cualificación profesional es innegable se mire por donde se mire.

Con carácter previo se ha querido dejar meridianamente clara una premisa que se considera de partida, basilar: el derecho a la formación de todas las personas trabajadoras y la importancia que la formación tiene en todos los sectores productivos y respecto de todas las ocupaciones y perfiles abarca también el empleo doméstico y conviene no olvidarlo y tenerlo muy presente.

El derecho a la formación como derecho laboral básico viene recogido tanto en los arts. 27, 35.1 y 40.2 CE como en el art. 4.2.b ET que garantizan, en general, tanto la educación y la formación profesional como la promoción a través del trabajo. En este ámbito que nos ocupa, el RD 1620/2011 que regula la relación laboral de carácter especial del servicio del hogar familiar (derogando su precedente inmediato, el RD 1424/1985), reconoce a las personas trabajadoras de este sector todos los derechos y deberes establecidos en los arts. 4-5 de la norma estatutaria. Por tanto, resulta meridiamente claro que el art 4.2.b) ET antedicho es de plena aplicación al empleo doméstico, sin especialidad o matización alguna.

Como acertadamente se ha señalado, «el derecho y el deber del trabajador de formarse profesionalmente debe asumirse como elemento esencial de la propia actividad laboral, con la influencia que ello debe tener sobre el régimen jurídico que posteriormente la regulación ordinaria decida otorgar a una $\mathrm{u}$ otra concepción de la actividad formativa. No obstante, el acto de formarse es actualmente para el trabajador un derecho y deber que, como ciudadano, le acompaña más que nunca en el marco de la relación laboral. En fin, las circunstancias que en el presente presiden las relaciones laborales españolas, caracterizadas por su elevada tasa de temporalidad y por su tendencia hacia la flexibilidad en todas sus variantes, invitan a robustecer la formación continua del trabajador dependiente y reconocer explícitamente en el marco constitucional la relevancia que ha adquirido la misma» (Requena, 2019: 151).

Y si se hace el ejercicio de extrapolar todo ello al ámbito que nos ocupa, resulta que para que los servicios tanto domésticos como de cuidados intrafamiliares en el domicilio sean eficaces, las trabajadoras del sector también deben mejorar tanto sus competencias genéricas como la garantía de calidad de los servicios prestados en este sector. El papel de la formación en este ámbito ha de ser capaz de identificar, partiendo de la gama de competencias que necesitan las personas trabajadoras, los programas de formación más adecua- 
dos y hacer las recomendaciones necesarias para mejorar la formación en el campo.

En el modelo que España ha adoptado, la cualificación profesional puede ser adquirida a través de diferentes itinerarios. En concreto, se reconocen tres vías para obtener cualificaciones profesionales:

a) La vía formal, superando los módulos formativos de los certificados de profesionalidad o de cualquier enseńanza regulada por el sistema educativo "con significación para el empleo»;

b) La vía de la experiencia laboral, a través del reconocimiento y la acreditación de la experiencia práctica de la actividad laboral, que puede implicar la exención total o parcial del módulo de Formación en el centro de trabajo, $y$;

c) Las vías no formales de adquisición de competencias, mediante el aprendizaje acumulado «a lo largo y ancho de la vida», como por ejemplo cursillos, seminarios, prácticas no laborales en empresas, etc.

Lo que promueve el marco normativo español de formación profesional, que se expondrá a continuación, es la clasificación de las cualificaciones profesionales "con arreglo a un catálogo que, organizado por módulos y ajustado a los requerimientos del sistema productivo, facilite la evaluación y acreditación de competencias profesionales, admitiendo que pueda alcanzarse el mismo resultado por otras vías menos estructuradas o formales (...). A partir de tal organización de la formación profesional se pretende promover el seguimiento y conjugación de las ofertas formativas de formación profesional, facilitándose su consecución mediante una articulación modular que permite la «acreditación parcial acumulable» de las cualificaciones adquiridas a través de cualquier vía» (Requena, 2019: 174-175).

En consecuencia, los instrumentos de acreditación y certificación oficial - con validez en todo el territorio nacional- de las cualificaciones y competencias profesionales contenidas en el Catálogo Nacional de Cualificaciones Profesionales $(\mathrm{CNCP})$ descansan sobre el siguiente tándem: por un lado, los títulos de formación profesional, que acreditan las enseñanzas de la formación profesional de ciclo largo o reglada, y, por otro lado, los certificados de profesionalidad, procedentes de la formación profesional de ciclo corto, entre la que destaca la formación profesional para el empleo, sin perjuicio de que puedan obtenerse estos certificados por otras vías. Pero veámoslo detenidamente. 


\section{2. ¿De qué formación se dispone? Una radiografía de la oferta formativa existente}

En el empleo doméstico, es frecuente que muchas personas trabajadoras pese a tener una dilatada experiencia profesional, no dispongan de la acreditación oficial necesaria para optar a determinados puestos de trabajo. Obvio es que cuando una persona dispone de estas competencias profesionales y es capaz de realizar correctamente las tareas propias de su puesto de trabajo, esté cualificada para ejercer su profesión, si bien, es frecuente, como decimos, que no suela contar con un documento oficial que lo reconozca y acredite como tal. Precisamente por ello, y como se verá a continuación, el establecimiento tanto de ciclos formativos de formación profesional inicial como de certificados de profesionalidad como documentos oficiales que acreditan la capacidad para ejercer esta actividad profesional viene a permitir (o ese es su leitmotiv) acceder en mejores condiciones a las ofertas de empleo del sector.

Como acertadamente se ha señalado, "la práctica que se adquiere mediante la formación profesional, modifica, moldea y enriquece la profesionalidad del trabajador, elemento de reveladora importancia en la relación de trabajo, puesto que resulta inseparable al carácter personalísimo de esta relación. A diferencia de la situación «tradicional», ahora se quiere cada vez más, «un profesional». En realidad, lo que esto parece indicar, es que la tendencia actual va en la dirección de seleccionar personas más productivas» (Sanz, 2019, 308). Pero en el empleo doméstico y a falta de la concreción de su contenido - ¿qué son tareas domésticas y de cuidados? - esto no ocurre, principalmente, como se ha tenido ocasión de adelantar, por su invisibilidad e infravaloración.

Cuesta comprender que siendo un lugar común pensar que disponer de competencias acordes con la demanda requerida de los puestos de trabajo continúa siendo la clave de bóveda en el éxito laboral, esto no sea así en el empleo doméstico, máxime la vertiginosa transformación que está teniendo en las últimas décadas como consecuencia de los cambios sociales anticipados. En los momentos que se viven, resulta paradójico que lo que cuenta cuando se contrata de una persona trabajadora de cualquier sector productivo, parece no contar para una trabajadora doméstica respecto de la que se pretende otorgue satisfacción inmediata a nuestras necesidades familiares sin preocuparnos por su formación/cualificación profesional en ese sentido y confiando en que cuenta con tales competencias por el simple hecho biológico de ser mujer. Por mucho que las actividades específicas a realizar — sean las que fueren a falta de concreción u objetivación - puedan realizarse de modo genérico, es evidente que se requiere de una básica formación/cualificación profesional para ello. Como en todas las ocupaciones, trabajos y actividades, cuanto mayor sea la técnica adquirida, mayor será la competencia y profesionalidad alcanzada en la prestación del servicio. 
Increíble tener que recordar esto. Y ello solo puede deberse a lo ya antedicho: la creencia de que el simple hecho biológico de ser mujer capacita de forma automática para la prestación de ciertas tareas como es el caso de las domésticas y de cuidados intrafamiliares.

Todo ello ha provocado que ante sectores (éste y otros) que presentan dificultades concretas de acreditación de competencias profesionales, se haya decidido regular títulos de formación profesional inicial (básicos) y certificados de formación profesional para el empleo (certificados de profesionalidad). Es importante tener en cuenta que tanto la formación profesional inicial como la formación profesional para el empleo son subsistemas de la Formación Profesional que, si bien dependen de ministerios distintos (Ministerio de Educación y de Empleo, respectivamente), están compuestas por cualificaciones profesionales modulares que permiten la convalidación entre ambos subsistemas ${ }^{3}$.

\subsection{La Formación Profesional Inicial específica: el ciclo formativo de formación profesional básica en actividades domésticas y limpieza de edificios}

El Catálogo Nacional de Cualificaciones Profesionales (CNCP), al que se ha hecho mención líneas atrás, es el instrumento del Sistema Nacional de las Cualificaciones y Formación Profesional (SNCFP) que ordena las cualificaciones profesionales susceptibles de reconocimiento y acreditación, identificadas en el sistema productivo en función de las competencias apropiadas para el ejercicio profesional. Comprende las cualificaciones profesionales más significativas del sistema productivo español, organizadas en familias profesionales y niveles. Constituye la base y el referente para elaborar la oferta formativa de los títulos de formación profesional y los certificados de profesionalidad. El CNCP incluye el contenido de la formación profesional asociada a cada cualificación, de acuerdo con una estructura de módulos formativos articulados. Y es el Instituto

3 La cualificación profesional describe un conjunto de competencias profesionales con significación en el empleo que pueden ser adquiridas mediante formación modular u otros tipos de formación, así como a través de la experiencia laboral (ex Ley Orgánica 5/2002, de 19 de junio, de las Cualificaciones y de la Formación Profesional). Este conjunto de competencias profesionales (conocimientos y capacidades) permite dar respuesta a ocupaciones y puestos de trabajo relevantes, con valor y reconocimiento en el mercado laboral. Se concreta en un documento que recoge de forma estructurada dichas competencias y una vez aprobado, se publica en el BOE en forma de Real Decreto. En ningún caso regula la profesión o actividad profesional, lo que corresponderá a las diferentes Administraciones competentes según el sector o ámbito de desarrollo. La cualificación no se corresponde con un plan de formación, sino que es la referencia, en el sistema integrado de formación profesional que describe la Ley Orgánica 5/2002, de 19 de junio, para que se elaboren los certificados de profesionalidad y los títulos de formación profesional. Cada cualificación se identifica mediante un código único que incluye la familia profesional a la que se asocia y su nivel [1, 2 o 3]. La competencia general define brevemente los cometidos y funciones esenciales del profesional. El entorno profesional describe el ámbito profesional en el que se desarrolla la actividad especificando el tipo de organizaciones, áreas o servicios; los sectores productivos, las ocupaciones y puestos de trabajo relacionados. 
Nacional de las Cualificaciones (INCUAL) el responsable de definir, elaborar y mantener actualizado el CNCP y el correspondiente Catálogo Modular de Formación Profesional.

Dicho esto, siendo la Ley Orgánica 5/2002, de 19 de junio, de las Cualificaciones y de la Formación Profesional el referente normativo de partida, como normativa de desarrollo en la materia es de destacar el Real Decreto $1128 / 2003$, de 5 de septiembre, por ser el encargado de regular dicho catálogo, si bien ha sido después modificado en varias ocasiones: el Real Decreto $1416 / 2005$, de 25 de noviembre, incorporó modificaciones puntuales; el Real Decreto 817/2014, de 26 de septiembre, vino a incorporar aspectos puntuales de las cualificaciones profesionales para cuya modificación, procedimiento de aprobación y efectos es de aplicación el artículo 7.3 de la Ley Orgánica 5/2002 antedicha; y varios Reales Decretos son los encargados de aprobar las diferentes cualificaciones profesionales. En suma, un sistema normativo prolijo y complejo en el que, en lo que a nuestro interés va referido, destaca el Real Decreto 1179/2008, de 11 de julio, por el que se complementa el CNCP, mediante el establecimiento de diecisiete cualificaciones profesionales de nivel 1, correspondientes a determinadas familias profesionales (anexo CDXIII), entre las que se encuentra la familia profesional de servicios socioculturales dentro de la que se ubica el trabajo doméstico y de cuidados. Este último, ha sido, a su vez, modificado por obra del Real Decreto 721/2011, de 20 de mayo, se establecen cuatro certificados de profesionalidad de la familia profesional servicios socioculturales y a la comunidad que se incluyen en el repertorio nacional de certificados de profesionalidad y se actualizan otros, entre ellos el de empleo doméstico.

La actualización de los currículos formativos de la formación profesional bá$\operatorname{sica}^{4}$ (en el ámbito de gestión del Ministerio de Educación) ha sido una constante para ir adaptándolos a las necesidades que de ellos se demandan, pero también la actualización de los certificados de profesionalidad de la formación profesional para el empleo. Las modificaciones/adaptaciones de ambos subsistemas han ido a la par, como no podía ser de otro modo, si bien las diferencias entre ambos son ostensibles en virtud de sus distintas filosofías formativas.

En cuanto a los primeros, esto es, los ciclos formativos de la formación profesional básica, el RD 774/2015, de 28 de agosto, estableció seis títulos entre los que se incluyó «Título profesional básico en actividades domésticas y limpieza de edificios» y cuyo currículo sería desarrollado por la Orden ECD/648/2016 que lo configura de la siguiente forma:

${ }^{4}$ Teniendo en cuenta el cambios introducido en la FP a través de la LOMCE (LO 8/2013, 9 de octubre, para la mejora de la calidad educativa), el RD 1147/2011 es el encargado de adaptarse a la nueva ordenación en esta área dando paso a la FP Básica tal y como hoy la conocemos. 


\section{Cuadro 1}

Orden ECD/648/2016, de 26 de abril, por la que se establece el currículo de seis ciclos formativos de formación profesional básica en el ámbito de gestión del Ministerio de Educación, Cultura y Deporte en BOE de 3 de mayo, pp. 29750-29751

Ciclo formativo de formación profesional básica en actividades domésticas y limpieza de edificios

\begin{tabular}{|c|c|c|c|c|}
\hline \multirow{2}{*}{ Módulos } & \multirow{2}{*}{$\begin{array}{c}\text { Duración } \\
\text { (horas) }\end{array}$} & \multirow{2}{*}{$\begin{array}{l}\text { Primer } \\
\text { curso } \\
\text { (h/s) }\end{array}$} & \multicolumn{2}{|c|}{ Segundo curso } \\
\hline & & & $(\mathrm{h} / \mathrm{s})$ & Horas \\
\hline 3098. Mantenimiento de prendas de vestir y ropa de hogar. & 275 & 7 & & \\
\hline 3102. Cocina doméstica. & 275 & 7 & & \\
\hline $\begin{array}{l}\text { 3145. Actividades de apoyo a personas no dependientes en } \\
\text { la unidad convivencial. }\end{array}$ & 130 & 4 & & \\
\hline 3009. Ciencias aplicadas I. & 160 & 5 & & \\
\hline 3011. Comunicación y sociedad I. & 160 & 5 & & \\
\hline Tutoría. & & 2 & & \\
\hline $\begin{array}{l}\text { 3104. Limpieza de domicilios particulares, edificios, oficinas } \\
\text { y locales. }\end{array}$ & 200 & & 8 & \\
\hline 3116. Limpieza con máquinas. & 80 & & 3 & \\
\hline 3146. Seguridad en el ámbito doméstico. & 130 & & 4 & \\
\hline 3042. Ciencias aplicadas II. & 160 & & 6 & \\
\hline 3012. Comunicación y sociedad II. & 190 & & 7 & \\
\hline Tutoria. & & & 2 & \\
\hline
\end{tabular}

\begin{tabular}{r|r|r|r|r}
\hline 3147. Formación en centros de trabajo. & 240 & & & 240 \\
\hline Total en el ciclo formativo $\ldots \ldots \ldots \ldots \ldots \ldots \ldots$ & 2000 & 30 & 30 & 240 \\
\hline
\end{tabular}

Se trata, por tanto, de un ciclo formativo de formación profesional básica o inicial que se presenta con una duración extensa (2.000 horas repartidas en dos cursos académicos) y con un vasto contenido (desde mantenimiento de ropa, cocina doméstica o cuidado de no dependientes pasando por ciencias aplicadas, comunicación y sociedad, seguridad en el ámbito doméstico o limpieza de máquinas). Un título que hace una oferta, interesante y completa que poco tiene que ver con los módulos formativos dentro de la formación profesional para el empleo que se centran en los contenidos más básicos de esta relación laboral pero que, obviamente, van dirigidos a colectivos distintos y con objetivos claramente diferentes: en general, el primero a jóvenes que optan por la formación profesional básica en lugar de la educación secundaria obligatoria; mientras el segundo a jóvenes y no tan jóvenes que, ante la falta de otras alternativas pueden decantarse por esta suerte de formación profesional para el empleo/acreditación de la experiencia laboral, como se verá a continuación. 
Y entre los variados y diversos contenidos de aprendizaje se encuentran desde limpiar, higienizar y desinfectar aseos, cocinas y estancias en domicilios particulares y edificios, organizar las actividades domésticas relacionadas con la alimentación en domicilios particulares o lavar, planchar, ordenar y coser la ropa del hogar y las prendas de vestir; pasando por otras como resolver problemas predecibles relacionados con su entorno físico, social personal y productivo, actuar de forma saludable en distintos contenidos cotidianos que favorecen el desarrollo personal y social o valorar las actuaciones encaminadas a la conservación del medio ambiente; y terminando por algunas referidas a cumplir las normas de calidad, las medidas de prevención de riesgos laborales ejercer sus derechos y cumplir sus obligaciones derivadas de su actividad profesional.

Superado el ciclo formativo de formación profesional básica referido se podrá obtener el certificado de profesionalidad en actividades domésticas y limpieza de edificios y que capacita para trabajar como:

— Limpiador doméstico / limpiadora doméstica.

- Cocinero doméstico / cocinera doméstica.

- Planchador doméstico / planchadora doméstica.

- Empleada / empleado de hogar.

— Limpiador / limpiadora.

— Peón especialista de limpieza.

- Especialista de limpieza.

— Limpiador / limpiadora de cristales.

El objetivo, por tanto, no es otro que formar profesionales que puedan ejercer su actividad por cuenta ajena tanto en domicilios particulares como en empresas públicas o privadas de cualquier tamaño. Además, podrán desarrollar su actividad profesional en el sector de la limpieza e higienización de toda clase de edificios, locales, centros o instituciones sanitarias y de salud, públicos o privados, industrias, elementos de transporte (terrestre, aéreo, marítimo), máquinas, espacios e instalaciones, soportes publicitarios y mobiliario urbano. Así como desempeñar sus funciones siguiendo instrucciones recibidas y bajo la supervisión directa de un responsable, pudiendo trabajar de forma autónoma o integrarse en un equipo de trabajo.

Y, además, permite el acceso, si fuera el caso, a título de formación profesional de grado medio, con preferencia en la admisión a todos los títulos de grado medio de las familias profesionales de Textil, Confección y Piel, Hostelería y Turismo y Servicios Socioculturales y a la Comunidad. Concretamente, una extensión formativa de continuidad bien pudiera ser la de Técnico en Atención a Personas en situación de dependencia destinado a capacitar a profesionales que ejerzan su actividad en el sector de servicios a las personas: asistenciales, psicosociales y de apoyo a la gestión doméstica y que certifica poder trabajar como: 
- Cuidador / cuidadora de personas en situación de dependencia en diferentes instituciones y / o domicilios.

- Gobernante / gobernanta y subgobernante / subgobernanta de personas en situación de dependencia en instituciones.

- Auxiliar responsable de planta de residencias de mayores y personas con discapacidad.

- Auxiliar de ayuda a domicilio.

- Asistente de atención domiciliaria.

— Trabajador / trabajadora familiar.

- Auxiliar de educación especial.

- Asistente personal.

— Teleoperador / teleoperadora de teleasistencia.

Sea como fuere, quiere adelantarse el escaso éxito de esta oferta formativa. Hoy por hoy solo se oferta en 18 centros, frente a los casi 450 que ofertan algunos de los ciclos de grado medio antedichos. Centros educativos y localidades que en la mayoría de las ocasiones nos sugieren zonas deprimidas, clase social baja y altas tasas de fracaso escolar, desempleo juvenil y de larga duración, con lo que resulta que la formación impartida también parece tener un cierto sesgo de clase social ${ }^{5}$.

\subsection{La Formación Profesional para el empleo específica: el certificado de profesional para el empleo doméstico}

La formación para el empleo es considerada un instrumento clave para reforzar la productividad y competitividad de las empresas españolas así como para acreditar la empleabilidad de las personas trabajadoras.

El desarrollo del derecho a la promoción y formación profesional en el trabajo reconocido en los arts. 4.2.b) y $23 \mathrm{ET}^{6}$ necesita ser concretado y, para ello, se remite prioritariamente a la negociación colectiva y, subsidiariamente, al acuerdo individual. Y, en este sentido, se ha señalado cómo «es, precisamente, en este ámbito (negociación colectiva) donde debiese producirse un ajuste más preciso e inmediato de la oferta y la demanda de competencias profesionales a partir de una detección adecuada de las necesidades formativas de las plantillas, de la planificación de la formación, y de la utili-

\footnotetext{
5 https://www.educacion.gob.es/centros/buscar.do?shortcut $=1 \&$ codaut $=00 \&$ codprov $=00 \&$ sse 1_natur $=0 \&$ comboniv $=141 \&$ combofam $=120123 \&$ textofamilia $=$ SERVICIOS $\% 20$ SOCIOCULTU RALES\%20Y\%20A\%20LA\%20COMUNIDAD\%20(LOE) \&comboens=12312301\&textoensenan $\mathrm{za}=$ Actividades\%20Dom\%e9sticas\%20y\%20Limpieza\%20de\%20Edificios

${ }^{6}$ Dentro de este subsistema de formación profesional, interesa diferenciar que hay una formación profesional obligatoria (ex lege y en supuestos como los relacionados con la prevención de riesgos laborales o las necesidades concretas de la empresa como la adaptación) y otra voluntaria a la que se dedica en exclusiva el art. 23 ET encargado de la formación programada dentro de la empresa.
} 
zación y gestión de los recursos disponibles en el sistema" (Nieto, 2018: 4). Dicho lo cual, en este sector en el que ni existe convenio colectivo ni se lo espera por lo que se dirá más delante, todo ello no hace sino poner en evidencia la gravedad de las dificultades formativas. Hay quienes se aventuran a hablar de «brecha de representación» (Gómez, 2020) en este sector en el que la escasa sindicación y la nula negociación colectiva en un país con elevada cobertura convencional están dificultando alcanzar los estándares de empleo decente que las instituciones mundiales han establecido como básicos y esenciales (Salcedo, 2020: 2).

El sistema de Formación Profesional para el empleo ha venido a desarrollarse por obra, primero, de la Ley 30/2015 y, después, del Real Decreto 694/2017. La referida Ley 30/2015 acomete una reforma integral del sistema de formación profesional para el empleo (en la línea predeterminada tanto por la Ley de Empleo 26/2003 como por el acuerdo sobre la Formación Profesional para el empleo de 2014 y el RDL 3/2011 en un intento por mejorar la empleabilidad de la clase trabajadora y conseguir que la Formación Profesional para el empleo (en adelante, FP) contribuya a superar los déficits formativos siempre denunciados y nunca removidos con éxito. Esta reforma integral se estructura en cuatro objetivos estratégicos: 1. La garantía del ejercicio del derecho a la formación de trabajadores, empleados y desempleados, en particular, de los más vulnerables; 2 . La contribución efectiva de la formación a la competitividad de las empresas: 3 . El fortalecimiento de la negociación colectiva en la adecuación de la oferta formativa a los requerimientos del sistema productivo, así como la eficiencia y transparencia en la gestión de los recursos públicos; y 4. Consolidar en el sistema productivo una cultura de formación profesional y favorecer con ello la creación de empleo estable y de calidad.

En desarrollo de la anterior norma legal, es, primero, el RD 34/2008 (18 de enero) el encargado de regular, en general, los certificados de profesionalidad y, después, el RD 1179/2008 (11 de julio) por el que se complementa el CNCP, mediante el establecimiento de diecisiete cualificaciones profesionales de nivel 1, correspondientes a determinadas familias profesionales, entre ellas la de servicios socioculturales en que se encuadra el empleo doméstico, el encargado de formular un sistema modular de 240 horas de duración -que es el que mejor se adecua a los intereses personales y laborales de los interesadas y permite diseñar su itinerario formativo tanto de forma presencial como a distancia en función de sus concretas necesidades- que incluye tres módulos formativos sobre acondicionamiento de camas y ropa, limpieza y cocina doméstica que, básicamente, capacitan o cualifican para dedicarse profesionalmente a la ocupación de limpiador/a, cocinero/a y planchador/a domésticos/as y, en definitiva, empleado/a de hogar (que aglutina todas las anteriores). 
Por tanto, España, como otros países de nuestro entorno comparado más cercano como es el caso de Francia ${ }^{7}$, ha considerado oportuno implementar un modelo que se sustenta sobre la base de la obtención de un título formativo básico/certificado de profesionalidad, según los casos, que aglutina los conocimientos, competencias ${ }^{8}$ y cualificaciones ${ }^{9}$ profesionales necesarias para realizar correctamente las tareas propias de esta actividad laboral. Por todos es sabido la importancia de las certificaciones oficiales en la era de la meritocracia que se vive $y$, precisamente por ello, se trata de un documento oficial otorgado por los servicios públicos de empleo autonómicos o estatal y que tiene validez en todo el territorio nacional.

En este segundo supuesto, el certificado de profesionalidad para el empleo doméstico comprende las acciones formativas dirigidas a la adquisición y mejora de las competencias y cualificaciones profesionales, tiene carácter modular con el fin de favorecer la acreditación parcial acumulable de la formación recibida y posibilitar al trabajador que avance en su itinerario de formación profesional para el empleo cualquiera que sea su situación laboral en cada momento y acredita una cualificación profesional del CNCP.

Solo repárese en que nada hay de cuidados, como sí se prevé en el ciclo formativo de la formación profesional inicial anteriormente analizado aunque sea a nivel muy básico, dado que esa formación tiene prevista impartirse en otro certificado de profesionalidad, este de nivel 2 sobre atención sociosanitaria de personas en el domicilio (RD 295/2004) cuyos contenidos pueden verse en el siguiente cuadro, pero que no hacen sino evidenciar como se mantiene el empeńo por separar tareas y cuidados intrafamiliares (excluyendo la atención especializada a las personas dependientes) como si ello fuera posible en la mayoría de los casos y reclamando con urgencia la objetivación del contenido que le es propio al empleo doméstico. Mientras no se acometa esa labor de determinación mate-

7 El país galo cuenta con 3 certificados profesionales, compuestos por 4 bloques comunes y 4 bloques específicos, certificables, todos ellos, separadamente y accesibles a través de la formación o de la validación de la experiencia profesional (casi 600 horas); más de 70 módulos de formación continua (entre 14 y 40 horas de duración) y un acompańamiento específico para la validación de la experiencia profesional que incluyen las tareas y los cuidados familiares (empleo doméstico, asistente de niños y asistente de dependientes). Más detalles en https://www.francecompetences.fr/

8 Es el conjunto de conocimientos, capacidades, habilidades y otros comportamientos que permiten ejercer de forma adecuada las tareas que se tienen que realizar en una determinada profesión. Las competencias indican la forma de comportarse o actuar en determinadas situaciones y están formadas por los conocimientos, las aptitudes y las actitudes y las capacidades.

9 Es un conjunto de competencias profesionales importantes para el empleo que pueden ser adquiridas mediante formación, o a través de la experiencia laboral. Acreditar las competencias profesionales contribuye al desarrollo personal y profesional, ya que permite: Obtener un documento oficial válido en todo el territorio espańol, que permite demostrar a una persona sus competencias profesionales; valorar la experiencia profesional; contar con más posibilidades para acceder a un empleo; y completar y mejorar la formación para aumentar las competencias profesionales. 
rial, será difícil que la formativa no solo acierte sino sea apetecible e interesante a las personas trabajadoras del sector que quieran formarse por atender a las necesidades que se les requieren. Este segundo certificado, el de atención sociosanitaria a personas en el domicilio, es más conocido y popular que el primero -el de empleo doméstico, prácticamente desconocido y difícil de conseguir-, se requiere para trabajar en empresas que ofrecen este servicio bajo cobertura púbica o institucional y que, precisamente por ello, es considerado socialmente como «un verdadero trabajo», «una verdadera profesión».

\section{Cuadro 2}

Real Decreto 721/2011, de 20 de mayo, por el que se establecen cuatro certificados de profesionalidad de la familia profesional Servicios socioculturales y a la comunidad que se incluyen en el Repertorio Nacional de certificados de profesionalidad y se actualizan los certificados de profesionalidad establecidos como anexo I y II en el Real

Decreto 1379/2008, de 1 de agosto, en BOE de 23 de junio, pp. 66305-66306

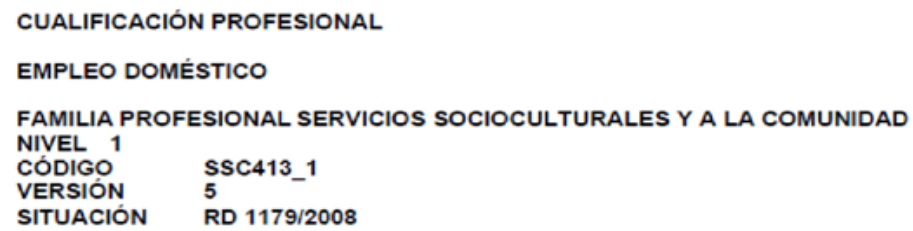

Realizar las tareas de limpieza de superficies y mobiliario de la vivienda, de elaboración de alimentos, de lavado y planchado, cosido básico manual y preparado de camas, seleccionando y empleando las técnicas, útiles, productos y electrodomésticos para garantizar la higienización del domicilio y permitir la disponibilidad de uso de las camas, la ropa de hogar, las prendas de vestir y el consumo de alimentos, cumpliendo con la normativa de prevención de riesgos laborales.

\section{UNIDADES DE COMPETENCIA}

UC1332_1: Efectuar el proceso de lavado, planchado y cosido básico manual de prendas de vestir y ropa de hogar, y la preparación de camas, en domicilio particular.

UC1330_1: Realizar la limpieza de domicilios particulares, en domicilio particular.

UC1331_1: Realizar el proceso de elaboración de alimentos en sus fases de compra, organización, manipulāción, cocinado y conservación, en domicilio particular.

\section{ENTORNO PROFESIONAL}

\section{Ámbito Profesional}

Desarrolla su actividad profesional tanto por cuenta propia como ajena en el ámbito público y privado. Así mismo, la persona profesional está capacitada para realizar la limpieza y preparación de domicilios particulares.

Sectores Productivos

Se ubica en el sector doméstico en las siguientes actividades productivas: limpieza y mantenimiento del domicilio, lavado, planchado, cosido básico manual de prendas de vestir y ropa de hogar, preparación de camas y compra y elaboración básica de alimentos.

Ocupaciones y puestos de trabajo relevantes

- Limpiador/a doméstico/a. Cocinero/a doméstico/a Planchador/a doméstico/a Empleado/a de hogar.

FORMACIÓN ASOCIADA (240 HORAS)

Módulos Formativos

MF1332_1: Acondicionamiento de camas, prendas de vestir y ropa de hogar.(90 h)

MF1330_1: Limpieza doméstica. $(60 \mathrm{~h})$

MF1331_1: Cocina doméstica.(90 h) 
Al margen del supuesto concreto de acceso al ciclo formativo antes analizado de la formación profesional básica, lo más habitual en la praxis, es que las personas trabajadoras en este sector se decanten (por razón de la edad, las dificultades de compatibilidad trabajo/estudios, etc.) bien por alcanzar este certificado profesional vía la realización de cursos de FP para el empleo ofertados por los servicios públicos de empleo estatal o autonómicos; bien por acceder al mismo vía la acreditación de la experiencia laboral o la formación no reglada que se tenga y, para ello, es preciso iniciar un procedimiento de reconocimiento, evaluación y acreditación de las competencias profesionales ${ }^{10}$. En estos casos, y dado que no haremos ninguna referencia posterior, quiere apuntarse que podrá obtenerse el certificado de profesionalidad vía el procedimiento de reconocimiento, evaluación y acreditación de las competencias profesionales que es convocado por las administraciones públicas competentes en función de las necesidades del mercado laboral y consiste en realizar las comprobaciones necesarias para determinar si una persona tiene las competencias necesarias para ejercer una determinada profesión. Para ello, se le pide a la persona que demuestre lo que sabe hacer, cómo realiza las tareas propias de un puesto de trabajo. Una vez superadas estas comprobaciones se puede obtener una acreditación oficial con validez en todo el territorio nacional.

Dicho esto, solo consta que se hayan hecho dos convocatorias entre los ańos 2018-2019 en la Comunidad Foral de Navarra, frente a las frecuentes convocatorias formuladas en todo el territorio nacional respecto de otras especialidades de la misma familia profesional (atención sociosanitaria a personas en el domicilio o atención sociosanitaria a personas dependientes en instituciones sociales) ${ }^{11}$. Lo que da mucho que pensar en la línea señalada tanto de falta de visibilización como de profesionalización de este sector.

${ }^{10}$ En este supuesto, hay tres tipos de requisitos (ex RD 1224/2009, de 17 de julio): edad, experiencia laboral y formación: tener 18 ańos cumplidos al realizar la inscripción si es para unidades de competencia de una cualificación profesional de nivel I o 20 ańos cumplidos al realizar la inscripción si es para unidades de competencia de cualificaciones profesionales de nivel II y III; contar con experiencia laboral adquirida en los últimos 10 años relacionada con las competencias que el candidato quiera acreditar de 2 años (1200 horas mínimas trabajadas) para cualificaciones de nivel I o de 3 años (2000 horas mínimas trabajadas) para cualificaciones de nivel II y III; y, por último, tener formación, realizada en los últimos 10 años también, relacionada con las competencias que el candidato quiera acreditar y, concretamente, 200 horas para cualificaciones de nivel I o 300 horas para cualificaciones de nivel II y III. rias.do

${ }^{11}$ https://sede.sepe.gob.es/recex/RXBuscadorConvocatoriaWebRED/busquedaConvocato- 


\section{3. ¿Por qué no se demuestra eficaz este sistema y quién es responsable de ello?: la importancia fundamental del asociacionismo y sindicación de las trabajadoras del sector}

Llegados a este punto y siendo una obviedad reconocer la mejora de las condiciones de trabajo de este sector que han contribuido a visibilizarlo, también es innegable que los problemas que persisten en materia de representación y negociación de este colectivo tienen por consecuencia que se haya conseguido mucho menos de lo deseado.

En otros sectores poco organizados como éste, el papel desempeñado por la ley ha sido fundamental tanto para reducir la desigual capacidad de negociación de trabajadores y empleados como para proteger a las personas trabajadoras. En Espańa, siendo innegable las positivas consecuencias derivadas de las reformas de 2011, los datos acumulados desde 2015 demuestran que, si en un primer momento aumentaron las afiliaciones, con posterioridad descendió el número de ocupadas, sin haber sido posible alcanzar las optimistas cifras de «afloramiento» previstas en aquellos momentos, sobre todo, por el incremento de los costes que la afiliación supone para los empleadores (las familias). Que ha habido aumento cuantitativo en el sector, lo avalan las cifras más recientes; pero no ha sido la esperada, ni mucho menos, máxime conociendo las cifras de trabajo informal existentes y que rondan el 70\% (EFSI, 2018: 22), informalidad que no es sino el síntoma de las limitaciones que existen respecto del marco legal vigente.

Siendo cierto y verdad que la aprobación de estas normas supuso en España un paso considerable en el afianzamiento de los derechos laborales de las personas trabajadoras de este sector, es fundamental tanto reducir las trabas administrativas que supone para familias y empleadas la oficialización de sus relaciones laborales como incentivar económicamente la suscripción de estas. Por tanto, remoción de trabas administrativas y acciones de estímulo económico público como ejes fundamentales del tránsito de la informalidad a la formalidad del sector. Pero, además, es crucial mantener su presencia en la agenda política para evitar posibles estancamientos y enfrentar los desafíos que aún quedan pendientes como la ratificación del Convenio 189 OIT. Y para ello resulta perentorio, además, dotarlas de mayor protección colectiva. Afortunadamente, desde hace un tiempo se vienen formando asociaciones de trabajadoras domésticas a fin de canalizar sus demandas y denuncias, y los sindicatos también les han dado cobertura, integrándolos en su estructura y promoviendo acciones para su promoción y apoyo. Es más, se dice que la no ratificación del Convenio 189 OIT está en el origen del movimiento asociativo reivindicativo. En otras palabras, la no ratificación del Convenio 189 OIT, asignatura pendiente para España y flagrante incumplimiento de sus compromisos internacionales, está funcionando, de facto, como el elemento actual de unidad fundamental del movimiento de 
defensa de los derechos de las trabajadoras domésticas que han centrado sus reivindicaciones en la necesidad de incorporar la protección por desempleo, pero también en la necesidad de fomentar, desde las instituciones públicas, el asociacionismo y sindicación de las trabajadoras domésticas o de promover organizaciones de empleadores e intermediarios con los que poder negociar sus condiciones laborales (Grau, 2019: 82 y ss.).

\subsection{Problemas de representación sindical y/o empresarial}

A nivel europeo, sólo en unos cuantos países, como Francia e Italia, existen federaciones de empleadores de trabajadores domésticos que permiten llevar a cabo negociaciones colectivas.

a) En Italia tres sindicatos negociaron un primer acuerdo colectivo con la Federación Nacional del Clero Italiano y dos asociaciones patronales/de empleadores en 1974. Durante las siguientes cuatro décadas los empleadores se organizaron para establecer sus propias organizaciones de representación: la Associazione Nazionale Famiglie Datori di Lavoro Domestico (DOMINA) y la Federazione Italiana Datori di Lavoro Domestico (FIDALDO). La FIDALDO firmó el correspondiente acuerdo en 1996 y la DOMINA lo hizo en 2001. El acuerdo vigente en la actualidad fue firmado en 2013 e incluye tasas de salarios, períodos de descanso, vacaciones pagadas, subsidio de enfermedad e indemnización por despido. El acuerdo carece de aplicación universal, ya que obliga únicamente a los empleadores miembros de la DOMINA o la FIDALDO, o a aquellos que firmaron contratos que remitieran explícita o implícitamente a los acuerdos de negociación. Cuando los trabajadores no cubiertos formalmente por estos contratos acuden a los tribunales, las disposiciones sobre salarios y seguridad social son las normas en que se basan los jueces para dictar sentencia. A pesar de las limitaciones mencionadas, en el sector del trabajo doméstico la situación ha mejorado significativamente en los últimos quince años y ha aumentado considerablemente el número de trabajadores que ha firmado contratos tipo.

b) En Francia, las relaciones triangulares han dado lugar a una serie de acuerdos colectivos con diferentes organizaciones de empleadores, que actuaban en representación de los cabeza de familia empleadores o de intermediarios y organismos (públicos o privados). El primero de estos acuerdos fue firmado en 1999 por las siguientes confederaciones sindicales: Fédération des particuliers employeurs de France (FEPEM), Confédération française démocratique du travail (CFDT), Confédération Générale du Travail (CGT), Confédération française des travailleurs chrétiens (CFTC) y Force Ouvrière (FO), y beneficiaba únicamente a trabajadores remunerados por particulares y empleados por los diferentes hogares. Se firmaron dos acuerdos más: 
uno en 2012, que firmaron seis sindicatos y dos organizaciones de empleadores, y beneficiaba a trabajadores domésticos colocados en viviendas de particulares por intermediarios sin fines lucrativos; y el segundo, firmado en 2014 por tres sindicatos y dos organizaciones de empleadores en representación de empresas privadas, que beneficiaba a trabajadores domésticos empleados por empresas privadas. Considerados conjuntamente, estos acuerdos abarcan la mayor parte del sector del trabajo doméstico que está cuantificado en 3.4 millones de empleadores particulares y 1.4 millones de trabajadores que generan 8.7 millones de euros en salarios pagados y 1.6 millones de horas declaradas (FEPEM, 2020: 6-7).

No cabe la menor duda que tanto la descentralización como el aislamiento en el que prestan sus servicios de las trabajadoras domésticas y el hecho de que, normalmente, no tengan compañeros y compañeras de trabajo, constituyen serias dificultades al ejercicio de sus derechos colectivos de asociación, representación y negociación colectiva. Cierto es que el ordenamiento jurídico español no lo prohíbe, pero sí que las «dificultades para su ejercicio, no han sido resueltas por la norma reglamentaria con una adaptación a las condiciones de los trabajadores de este sector» (Sanz, 2019a: 19). Y precisamente por ello es necesario aumentar la implicación política en este sentido de cara a lograr un mayor peso colectivo que les dé más fuerza, alentando derechos cruciales como el de la negociación colectiva con una incidencia directa en otros como el de formación. Y es que, por seguir en el modelo francés, dentro del sector existen 2 ramos profesionales con sendos acuerdos colectivos, fruto de un diálogo social muy positivo para ambas partes: trabajadores de empleadores particulares que regula tres perfiles profesionales -empleados domésticos, asistentes de dependientes y de niños ${ }^{12}$ - y asistentes infantiles de empleadores particulares con un único perfil profesional, esto es, el de asistentes infantiles que cuidan niños en sus domicilios y no en el de los niños, previa autorización de la administración competente y sujeta a un máximo de niños, un mínimo de espacio, medidas de seguridad, etc. ${ }^{13}$.

Se considera que, sin desmerecer otro tipo de organizaciones, son los sindicatos «los sujetos colectivos que deberían asumir prioritariamente esta importante función, por ser estructuras ya existentes, por la relevancia que se les reconoce, por las funciones que están llamados a desarrollar y por tener capacidad de unificar las reivindicaciones de los trabajadores del sector, y por tanto, de actuar

12 Convention collective nationale des salariés du particulier employeur du 24 novembre 1999, disponible enhttps://www.legifrance.gouv.fr/conv_coll/id/KALITEXT000005672603?tab_selection =all\&searchField=ALL\&query=Convention+collective+nationale+des+salaries $+\mathrm{du}+$ particulier+emplo yeur\&page $=1$ \&init $=$ true

13 Convention collective nationale des assistants maternels du particulier employeur du ler juillet 2004. Etendue par arrêté du 17 décembre 2004 JORF 28 décembre 2004, disponible en https:// www.legifrance.gouv.fr/conv_coll/id/KALICONT000005635807/ 
con mayor fuerza colectiva» (Miñarro, 2014: 87). Pero, donde no los haya, también cabe que se organicen a través de cooperativas o asociaciones. $Y$ en nuestro país, ambas posibilidades (sindicación ${ }^{14}$ y asociacionismo ${ }^{15}$ ) son factibles con los mimbres actuales y el marco jurídico establecido que equipara (por lo menos a nivel laboral) este colectivo al resto de trabajadores por cuenta ajena ${ }^{16}$. Y es que para que la negociación colectiva sea un hecho, las leyes y políticas deben contemplar la posibilidad de proceder a negociaciones colectivas sectoriales en las que queden representados las trabajadoras domésticas y las organizaciones de sus empleadores (OIT, 2016). Cuando ha habido voluntad política para solucionar problemas operativos como los apuntados, se han encontrado soluciones. Según algunos ejemplos aportados por la OIT, las organizaciones de amas de casa preexistentes se han reconocido cada vez más como empleadoras a la hora de negociar en países como Alemania y Uruguay.

\subsection{Problemas derivados de la prioridad de otros aspectos diferentes a los formativos que se consideran preferentes: prevención de riesgos laborales y protección por desempleo}

En la actualidad, es innegable la existencia en nuestro país una relativa preocupación sindical por este colectivo si bien la formación no está entre los aspectos fundamentales que marcan la acción sindical. De forma absolutamente lógica, entre las reivindicaciones sindicales enmarcables en este sector se encuentra la ratificación del convenio n. ${ }^{\circ} 189$ OIT, la urgente integración de este colectivo en el ámbito subjetivo de aplicación de la Ley de prevención de riesgos laborales (LPRL) y equiparación definitiva en materia tanto laboral como de seguridad social con la ampliación de la protección social hacia el desempleo como se ha puesto de manifiesto durante la pandemia del COVID-19.

Y es que, como se ha señalado, «en el plano de las resistencias, más costosas de superar por lo que suponen de oposición o protesta frente al cambio que suponen, es posible encontrar dos principales: la referida a la exclusión del trabajo doméstico de la aplicación de la Ley de Prevención de Riesgos La-

14 Pese a tratarse de un movimiento incipientes son ya dos los sindicatos específicos Sindicato de Trabajadoras del Hogar y los Cuidados (SINTRAHOCU, 2020) y SINDIHOGAR (2011), lo que pone de manifiesto las sinergias e inquietudes existentes a respecto.

15 También hay algunos referentes de ámbitos más limitados pero que están en la génesis del movimiento sindical antedicho: asociación de trabajadoras/es del hogar de Sevilla, asociación de trabajadoras del hogar de Bilbao (ATH-ELE), asociación Servicio doméstico activo (SEDOAC, 2005) que cuenta con un centro de atención integral y una red de apoyo al colectivo d dimensión nacional, entre otras.

16 Téngase en cuenta que el Convenio OIT n. ${ }^{\circ} 163$ sobre negociación colectiva (1981) garantiza que las partes en la negociación colectiva deberían estar en condiciones de elegir los niveles más apropiados de negociación colectiva y, en caso de ser necesario, se deberían adoptar las medidas adecuadas a las condiciones nacionales para que ésta pueda desarrollarse en cualquier nivel y especialmente, a nivel «del establecimiento, de la empresa, de la rama de actividad, de la industria y a nivel regional o nacional (art. 4.1) 
borales y de la protección por desempleo. Sendas exclusiones representan una merma de considerable repercusión en el marco protector del trabajo doméstico y se encuentran en el epicentro de todos los problemas de ratificación» (Grau, 2019: 79 y ss.).

Dicho lo cual, no está tan presente como debiera entre las preocupaciones sindicales la formación como elemento de dignificación, de valoración decisiva en este sector productivo y respecto del colectivo concreto que estamos tratando. Se considera un hecho de menor importancia frente a otras preocupaciones sindicales de mayor calado que por ir referidas a las idiosincrásicas condiciones de trabajo tienen un mayor protagonismo o repercusión. La formación en España, históricamente, ha ido a la zaga en casi todos los sectores productivos, incluso en aquellos en los que la formación va ligada a la prevención de riesgos laborales y, por tanto, a la seguridad y salud de las personas trabajadoras. Lo que no quiere decir que esté absolutamente ausente en la acción sindical, sería injusto ser tan tajante, pero se echa en falta una línea concreta referida a la formación y cualificación.

Repárese en lo llamativo de ejemplos como el francés donde desde la primera hora trabajada en el sector del empleo doméstico se tiene derecho a 58 horas de formación anuales gratuitas (financiadas con cargo al plan de desarrollo de competencias ${ }^{17}$ ) y el amplísimo elenco de incentivos fiscales a las familias por la contratación de este personal (hasta el 50\% del gasto anual en servicio doméstico de las familias con el tope máximo de 7.500 euros/año). La experiencia francesa respecto de la lucha contra la informalidad a través de contratos tipo $\mathrm{y}$ "cheques de empleo y servicios» ${ }^{18}$ o "ces $^{19}{ }^{19}$ ( (Quintero, 2020:61 y ss.) es una buena práctica que comienza a ensayarse en otros países europeos (Italia con el libretto famiglia ${ }^{20}$ ) y que podría ser interesante implementar en Espańa. El razonamiento es lógico: si las empresas/los empresarios cuentan con una serie de gastos deducibles que les permites llevar a cabo ciertas desgravaciones fiscales (con ciertos topes y limites) en sus impuestos, por qué no las familias que actúan como empleadores en estos casos?. Repárese en el hecho de que en nuestro país

17 A partir del 1 de enero de 2019, el plan de desarrollo de competencias del Ministerio de Trabajo, Empleo e Inserción francés (plan de développement des compétences) reemplaza a su predecesor inmediato el plan de capacitación. Este plan permite a los empleados seguir acciones formativas por iniciativa de su empleador, frente a formaciones que pueden seguir por iniciativa propia a través de su cuenta de formación personal. El empresario tiene dos obligaciones en materia de formación profesional: adaptación al puesto de trabajo y retención de empleados; también puede proponer acciones que contribuyan al desarrollo de competencias (artículo L 6321-1 del código de trabajo francés). Más detalles en https://travail-emploi.gouv.fr/formation-professionnelle/entreprise-et-formation/article/plan-de-developpement-des-competences

18 Que simplifica las formalidades administrativas para los particulares hasta el punto de que no es necesario que se registren en el organismo recaudador de los cuotas a la Seguridad Social ni emitir nóminas, entre otros.

19 https://www.cesu.urssaf.fr/info/accueil.html

${ }^{20}$ Más información en https://www.inps.it/nuovoportaleinps/default.aspx?itemdir=51098. 
esto resulte hoy por hoy impensable bajo el pertinaz argumento de la sospecha de fraude que estas relaciones laborales suscitan (contratación entre familiares y retribución del trabajo doméstico ejercido por «amas de casa»).

Esto es contradictorio, choca cuando tomamos conciencia de los beneficios que la formación incorpora, de forma casi automática, a la prestación de servicios o actividades. Y es que aumentan las oportunidades, mejoran las condiciones laborales, incrementan la satisfacción personal de las personas trabajadoras por lo que suponen de superación profesional. Cierto es que lo que la realidad arroja es un gran número de dificultades que se traducen tanto en lo referido a la oferta formativa como a la demanda. Por el lado de la oferta, es urgente que sindicatos, asociaciones y cooperativas tanto por el lado de las personas trabajadoras como de los empleadores se impliquen en la mejora de la formación, si bien son constantes las quejas respecto de dificultades como las referidas a la difícil compatibilidad horaria de la oferta formativa con el trabajo doméstica, así como el desconocimiento de las familias empleadoras de los derechos de sus trabajadoras en este sentido. Por el lado de la demanda, el colectivo de trabajadoras domésticas, en sentido lato, necesita que la información le llegue a través de los agentes antedichos por tratarse de un colectivo que, como se ha adelantado, está aislado. Pero también, es preciso formular una oferta formativa en términos lo suficientemente atractivos para que decidan, desde su libertad individual, invertir tiempo en ello. Y esto, normalmente, lo hacen para salir del círculo vicioso del empleo doméstico - sector refugio ante contingencias como las crisis económicas y la escasez de empleo en otros sectores- y redirigirse hacia sectores próximos como el de cuidados a dependientes -sector de tránsito hacia otros considerados mejores-. Fundamental, por tanto, invertir recursos en campañas de sensibilización, de asesoramiento colectivo o personalizado, programas específicos de educación y capacitación, etc., incluso en aquellos destinados a hacer atractiva la implicación por parte de las familias (cheques, incentivos, etc.). La necesidad de intervención estatal de fomento o incentivo en este sentido es ineludible.

Antes de concluir, resulta fundamental, en la misma línea, hacer mención somera a la iniciativa concreta que supone el Proyecto Prodome ${ }^{21}$ que persigue la profesionalización del trabajo doméstico en consonancia con el Marco Europeo de Cualificaciones (MEC) y que propone un curriculum europeo común para el sector, en definitiva, una certificación europea. Una muestra del interés que el tema analizado tiene y del afán europeo por hacer algo en favor de tal objetivo. Y una apuesta decidida sustentada en el convencimiento de que a mayor formación y cualificación, mayor será la mejora tanto en la calidad de los servicios como en los derechos de las personas trabajadoras. Y la única receta eficaz para salir del círculo perverso informalidad-desprofesionalización-desprotección.

${ }^{21}$ Más detalles al respecto disponibles en https://www.prodome.eu/es/los-resultados/ 


\section{Bibliografía}

EFSI, PHS Industry Monitor, Statistical overview of the personal and household services secteur in the European Union, April 2018, disponible en http://www.efsi-europe.eu/ fileadmin/MEDIA/publications/2018/PHS_Industry_monitor_April_2018.pdf

FEPEM (2020) Le secteur des particuliers employeurs et de l'emploi à domicile. Rapport sectoriel des branches, disponible en https://www.fepem.fr/wp-content/uploads/Rapportsectoriel-des-Branches_2020_VF.pdf

Gómez Abelleira, F. Javier (2020) «El trabajo al servicio del hogar: algunas propuestas desde el proyecto europeo PHS-QUALITY», 10.07.2020. Disponible en https://forodelabos.blogspot.com/2020/07/el-trabajo-al-servicio-del-hogar.html

Grau Pineda, Carmen (2019) «De sirvientas a trabajadoras: la necesaria ratificación del Convenio 189 OIT sobre trabajo decente para las trabajadoras y los trabajadores domésticos», Revista Jurídica de los Derechos Sociales Lex Social, vol. 9, n. ${ }^{\circ} 2$.

Grau Pineda, Carmen (2020) «Empleo doméstico y cuidadores informales: obstáculos para conciliar», en VVAA, Reflexiones sobre el empleo doméstico. De dónde venimos, dónde nos encontramos y hacia dónde vamos, Servicio Central de Publicaciones del Gobierno Vasco, 2020.

Miñarro Yanini, Margarita (2014) «Formas esclavas de trabajo y servicio del hogar familiar: delimitación conceptual, problemática específica y propuestas», Relaciones Laborales, n. ${ }^{\circ} 10$.

Nieto Rojas, Patricia (2018) «El nuevo Sistema de Formación Profesional para el empleo (Ley 30/2015 y RD 694/2017) y el papel de los agentes sociales en su desarrollo», Revista de Información Laboral, n. ${ }^{\circ} 7$.

OIT (2016) Voz y representación de los trabajadores domésticos a través de la organización, Nota e información n. ${ }^{\circ}$ 8, Servicio de Mercados Laborales Inclusivos, Relaciones Laborales y Condiciones de Trabajo (INWORK), Ginebra.

Quintero Lima, M. ${ }^{a}$. Gema (2020) «El cheque servicio como elemento de gestión/control del trabajo doméstico: la necesaria definición conceptual», en VVAA, Reflexiones sobre el empleo doméstico. De dónde venimos, dónde nos encontramos y hacia dónde vamos, Servicio Central de Publicaciones del Gobierno Vasco.

Requena Montes, Oscar (2019) Formación profesional continua y prestación de trabajo: El artículo 23 del Estatuto de los Trabajadores como mecanismo de conciliación, Tesis doctoral, disponible en acceso abierto en TESEO.

Salcedo Beltrán, Carmen (2020) «El trabajo doméstico en España: planteamientos y desafíos insoslayables frente a la "esclavitud moderna», Revista General de DTSS, Iustel, n. ${ }^{\circ} 55$.

SAnz SÁEZ, Concha (2019) «Derechos colectivos y empleadas del hogar: reformas jurídicas inaplazables para un trabajo decente del trabajo doméstico en España», comunicación presentada y seleccionada respecto de la conversación II «Trabajo decente para todos» en el Congreso Interuniversitario OIT sobre el futuro del trabajo, Sevilla, 7-8 febrero 2019.

SANZ SÁEz, Concha (2019) «Profesionalización efectiva del servicio doméstico. Un trabajo pendiente», Revista jurídica de los Derechos Social Lex Social, vol. 9, n.o 2/2019. 